\title{
In Vivo Angiogenic Activity and Hypoxia Induction of Heterodimers of Placenta Growth Factor/Vascular Endothelial Growth Factor
}

\author{
Yihai Cao, Philip Linden, David Shima, Fiona Browne, and Judah Folkman \\ Department of Surgery and Department of Cellular Biology, Harvard Medical School; and Department of Surgery, \\ Children's Hospital, Boston, Massachusetts 02115
}

\begin{abstract}
To investigate the in vivo angiogenic activity of placenta growth factor (PIGF) and its heterodimers with vascular endothelial growth factor (VEGF), the induction of neovascularization of these factors in the mouse cornea was studied. VEGF $_{165}$ is sufficiently potent to stimulate new capillary growth from the limbal vessels. $\mathrm{PlGF}_{129} / \mathrm{VEGF}_{165}$ heterodimers also induce corneal neovascularization with a maximal vessel length similar to $\mathrm{VEGF}_{165}$, but with a marked decrease of vessel density. In contrast, $\mathrm{PlGF}_{129}$ has little or no effect in this in vivo angiogenesis assay. The expression of VEGF mRNA and protein is drastically up-regulated by hypoxia in choriocarcinoma cells, whereas expression of PIGF is not affected by the low concentration of oxygen. Up-regulation of VEGF production results in increased formation of PIGF/VEGF heterodimers in these tumor cells. Thus, hypoxia indirectly up-regulates expression levels of PIGF/ VEGF heterodimers and modulates VEGF activity when these factors are co-expressed. (J. Clin. Invest. 1996. 98: 2507-2511) Key words: angiogenesis • placental growth factor • vascular endothelial growth factor
\end{abstract}

\section{Introduction}

The growth of new blood vessels, neovascularization, includes angiogenesis and vasculogenesis. Angiogenesis is essential for development, tissue regeneration, and remodeling (1). Neovascularization also plays a key role in pathologic conditions including tumor growth and metastasis, diabetic retinopathy, rheumatoid arthritis, psoriasis, and cardiovascular diseases $(2,3)$. In response to angiogenic stimuli, endothelial cells are able to change their morphology, cause vascular dilation, degrade the basement membrane, proliferate and migrate, and form capillaries.

The switch to angiogenesis is regulated by a local change of the equilibrium between angiogenic factors and inhibitors (2). Vascular endothelial growth factor (VEGF), ${ }^{1}$ also known as

Address correspondence to Yihai Cao, Department of Cell and Molecular Biology, Medical Nobel Institute, Karolinska Institute, S-171 77, Stockholm, Sweden. Phone: 46-8-7287300; FAX: 46-8-301833.

Received for publication 1 July 1996 and accepted in revised form 11 September 1996.

1. Abbreviations used in this paper: PlGF, placenta growth factor; VEGF, vascular endothelial growth factor.

J. Clin. Invest.

(C) The American Society for Clinical Investigation, Inc.

0021-9738/96/12/2507/05 \$2.00

Volume 98, Number 11, December 1996, 2507-2511 vascular permeability factor (VPF), is an endothelial cell-specific growth factor which plays a crucial role in regulation of endothelial growth, differentiation and maintenance of vascular integrity (4-9). The expression of VEGF can be up-regulated by hypoxia and other factors such as transforming growth factor and phorbol ester (10-12). The angiogenic effect of VEGF is mediated by two endothelial cell-specific receptor tyrosine kinases, Flk-1/KDR and Flt-1 $(12,13)$. In addition, three other endothelial cell specific tyrosine kinase receptors, Flt-4, tie-1 and tie-2/tek have been discovered $(12,14)$. Inactivation of VEGF, Flt-1, Flk-1, tie-1, and tie-2 genes by targeted mutations has shown that they are indispensable during the vascular development of the embryo (15-19).

Recently, three endothelial growth polypeptides with structural similarities to VEGF have been isolated (20-22). PlGF is found to be specifically expressed in the placenta and in some tumor cell types (23). PlGF forms natural heterodimers with VEGF when these two factors are coexpressed $(24,25)$. It binds with a high affinity to Flt-1 receptor, but not to Flk-1/ KDR (26). Vascular endothelial growth factor-B (VEGF-B), a nonglycosylated highly basic heparin-binding polypeptide, is a mitogen for endothelial cells and its expression is particularly abundant in cardiac and skeletal muscles. VEGF-B also heterodimerizes with VEGF in cells coexpressing both factors (21). Vascular endothelial growth factor-C (VEGF-C) is homologous to other members of VEGF and is the ligand for Flt-4 receptor, whose expression is restricted mainly to lymphatic endothelia (22). It stimulates the migration of capillary endothelial cells in vitro.

Although these homo- and heterodimeric angiogenic growth factors stimulate endothelial cell proliferation and migration in vitro, their in vivo biological functions remain uncharacterized. In this study, we report the in vivo angiogenic activity of $\mathrm{PlGF}_{129}$ and $\mathrm{PlGF}_{129} / \mathrm{VEGF}_{165}$ heterodimers, and the role of hypoxia in the regulation of heterodimerization between PIGF and VEGF in tumor cells.

\section{Methods}

Reagents, cells, and animals. Recombinant human $\mathrm{VEGF}_{165}, \mathrm{PlGF}_{129}$ and $\mathrm{PlGF}_{129} / \mathrm{VEGF}_{165}$ heterodimers were prepared as previously described (24). These dimeric growth factors were purified to homogeneity using antibody-coupled affinity chromatography (24). Human choriocarcinoma cell lines, JAR and JE-3, were obtained from the American Type Culture Collection (ATCC) and maintained in DME supplemented with $10 \%$ FCS (Hyclone, Logan, UT) and antibiotics. Male 5-6-wk-old C57B16/J mice (The Jackson Laboratory, Bar Harbor, ME) were acclimated and caged in groups of four or less. Animals were anesthetized in a methoxyflurane (Pitman-Moore Inc., Mundelein, IL) chamber before all procedures. Mice were killed with a lethal dose of methoxyflurane. All animal studies were reviewed and approved by the animal care and use committee of Children's Hospital, Boston and are in accordance with the guidelines of the Department of Health and Human Services. 
Hypoxia treatment and Northern blot analysis. Monolayers of human choriocarcinoma (JAR and JE-3) and hepatoma (Hep G2) cells were grown in $25-\mathrm{cm}^{2}$ flasks to confluency in DMEM supplemented with $10 \%$ FCS. Cells were then incubated at $37^{\circ} \mathrm{C}$ in a normoxia $(21 \%$ $\mathrm{O}_{2}$ and $\left.10 \% \mathrm{CO}_{2}\right)$ or a hypoxia $\left(2 \% \mathrm{O}_{2}, 10 \% \mathrm{CO}_{2}\right)$ chamber for $18 \mathrm{~h}$. After incubation, total RNA was extracted by a modified acid phenol/guanidine method as previously described (11). $15 \mu \mathrm{g}$ of total RNA were separated by $1 \%$ agarose-formaldehyde gel, transferred by Northern capillary blot onto a Gene-Screen Plus membrane (DuPont-NEN, Boston, MA), fixed by UV cross-linking and hybridized to a cDNA probe of PlGF-2 (PlGF 150$)$. The cDNA coding for PlGF-2 was cloned into a T-A plasmid vector (Invitrogen Crop., San Diego, CA) by a PCR method using human placenta cDNA as the template. The intact insert for PlGF-2 was sequenced, isolated and used as the probe for hybridization. The human $\mathrm{VEGF}_{165} \mathrm{cDNA}$ probe was labeled using a random priming method as previously described (11).
Mouse corneal micropocket assay. The mouse micropocket corneal assay was carried out according to procedures previously described $(27,31)$. Male 5-6-wk-old C57B16/J mice were used. Corneal micropockets were created with a modified von Craefe cataract knife in both eyes of each mice. Into each pocket, a pellet $(0.35 \times 0.35 \mathrm{~mm})$ of sucrose aluminum sulfate (Bukh Meditec, Copenhagen, Denmark) coated with hydron polymer type NCC (IFN Sciences, New Brunswick, NJ) containing $160 \mathrm{ng}$ of $\mathrm{PlGF}_{129}, \mathrm{VEGF}_{165}$ or $\mathrm{PlGF}_{129} / \mathrm{VEGF}_{165}$ heterodimers was implanted. The pellet was positioned $0.6-0.8 \mathrm{~mm}$ from the corneal limbus. After implantation, erythromycin ophthalmic ointment (E. Fougera, Melville, NY) was applied to each eye. The corneas with implanted pellets containing growth factors were examined by a slit-lamp biomicroscope on day 5 after pellet implantation. Vessel length and clock hours of circumferential neovascularization were measured.

ELISA immunoassays. Monolayers of JE-3 choriocarcinoma cells

\section{A No Factor}

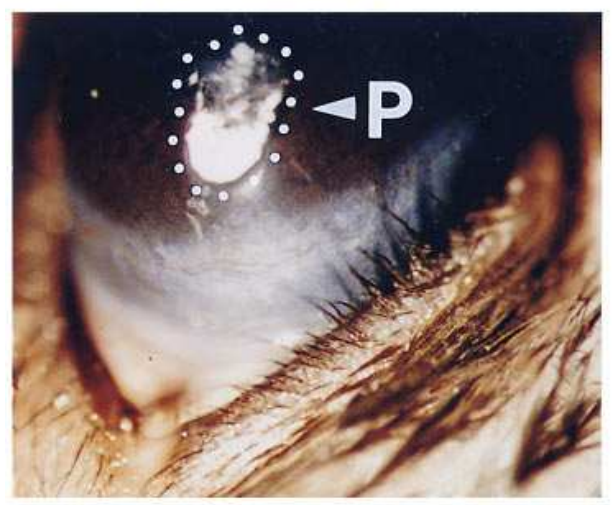

C VEGF/PLGF
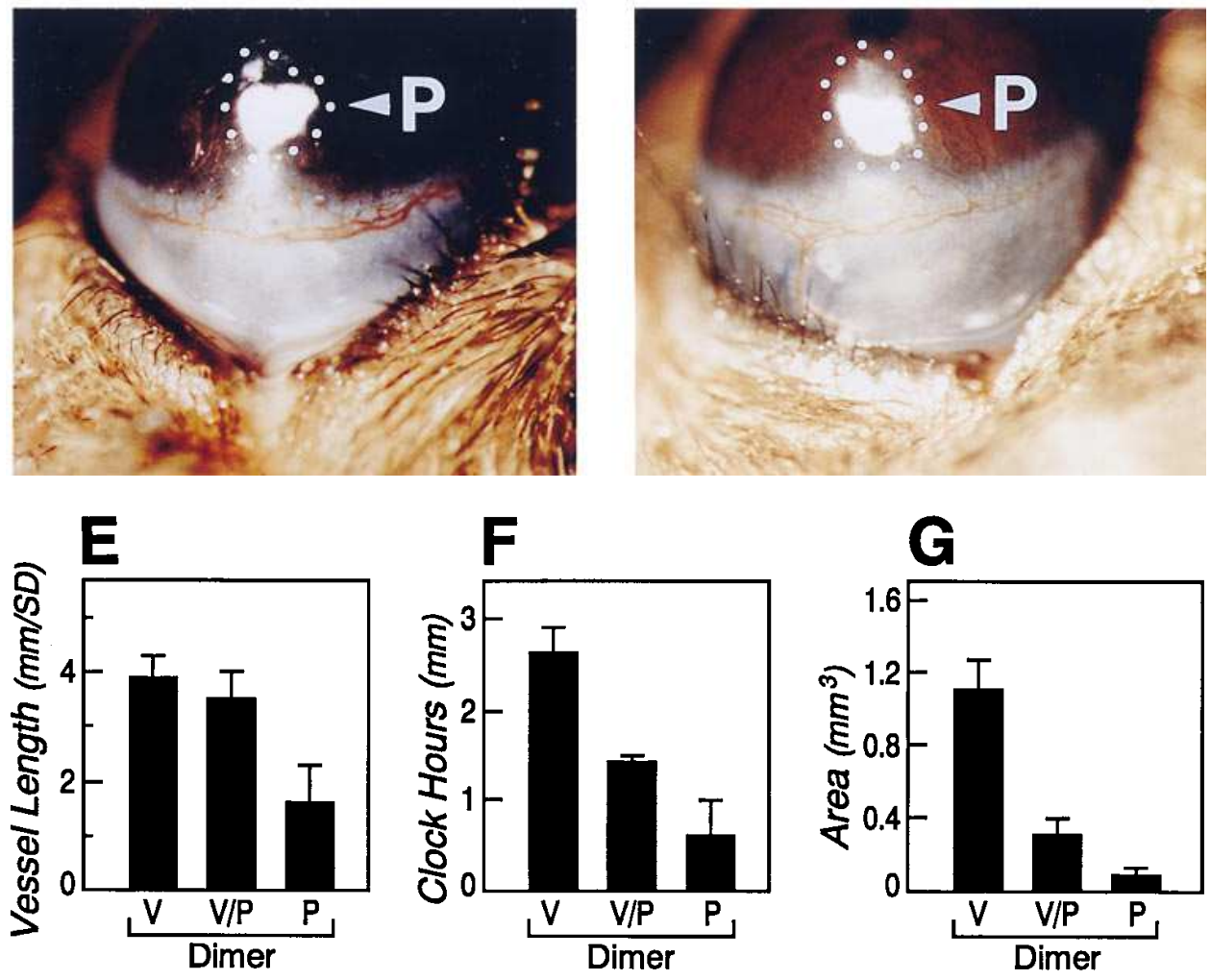

Figure 1. Stimulation of mouse corneal neovascularization by $\mathrm{PlGF}_{129}$, $\mathrm{VEGF}_{165}$, and $\mathrm{PlGF}_{129} / \mathrm{VEGF}_{165}$. Pellets of sucrose aluminum sulfate coated with hydron containing 160 ng of $\mathrm{VEGF}_{165}$ homodimers $(B)$, $\mathrm{PlGF}_{129} / \mathrm{VEGF}_{165}$ heterodimers $(C)$, or PlGF $_{129}$ homodimers $(D)$ were implanted into corneal micropockets of C57B16/J mice as described in Methods. Corneas were photographed by slit-lamp stereomicroscopy on day 5 after growth factor implantation. Photographs represent $\sim 20 \times$ amplification of the mouse eye. $(A)$ Pellets without growth factors were used as controls. Dots encircle the area of the implanted pellet. $P$, pellet. $(E-G)$ Quantitation of corneal neovascularization was performed on day 5 after pellet implantation. $(E)$ Maximal vessel length. $(F)$ Clock hours of circumferential neovascularization. $(G)$ Areas of neovascularization. $V, \mathrm{VEGF}_{165}$ homodimers; $V / P$, $\mathrm{VEGF}_{165} / \mathrm{PlGF}_{129}$ heterodimers; $P$, PlGF $_{129}$ homodimers. 
were grown to confluency in DME supplemented with $10 \%$ FCS in $75-\mathrm{cm}^{2}$ flasks. The medium was replaced with fresh DMEM containing $10 \%$ FCS and 10 units $/ \mathrm{ml}$ of heparin (Abbott Laboratories, Chicago, IL). Cells were then incubated at $37^{\circ} \mathrm{C}$ in a normoxia $\left(21 \% \mathrm{O}_{2}\right.$ and $\left.10 \% \mathrm{CO}_{2}\right)$ or a hypoxia $\left(2 \% \mathrm{O}_{2}, 10 \% \mathrm{CO}_{2}\right)$ chamber for $48 \mathrm{~h}$. The conditioned medium was used to quantitatively measure PlGF, VEGF and PlGF/VEGF heterodimers. "Sandwich" enzyme detection methods using specific anti-VEGF and anti-PIGF antibodies were used as previously described (24).

\section{Results}

Stimulation of mouse corneal neovascularization. Pellets of sucrose aluminum sulfate containing the slow release polymer hydron and the dimers of $\mathrm{PlGF}_{129}, \mathrm{VEGF}_{165}$, and $\mathrm{PlGF}_{129}$ / $\mathrm{VEGF}_{165}$ were surgically implanted into the micropockets of mouse corneas (Fig. 1). Stimulation of circumferential neovascularization from the limbal vessels was examined on day 5 after implantation. The angiogenic response of corneas induced by $160 \mathrm{ng}$ of $\mathrm{VEGF}_{165}$ homodimers was intense with a high density of new capillaries and a marked limbal vessel dilation (Fig. $1 \mathrm{~B}$ ). Similar to $\mathrm{VEGF}_{165}$, the same amount of $\mathrm{PlGF}_{129} /$ VEGF $_{165}$ heterodimers was also sufficiently potent to stimulate the outgrowth of capillaries from the limbal vessels (Fig. 1 $C)$. The capillary vessel length of $\sim 0.4 \mathrm{~mm}$ was virtually indistinguishable in corneas implanted with $\mathrm{VEGF}_{165}$ and $\mathrm{PlGF}_{129} /$ VEGF $_{165}$ heterodimers (Fig. $1, B, C$, and $E$ ). However, a dramatic difference of vessel density and clock hours was observed between $\mathrm{PlGF}_{129} / \mathrm{VEGF}_{165}$ heterodimer- and $\mathrm{VEGF}_{165}$ homodimer-implanted corneas (Fig. $1, B, C$, and $F$ ). In contrast to $\mathrm{VEGF}_{165}$ and $\mathrm{PlGF}_{129} / \mathrm{VEGF}_{165}$ heterodimers, $\mathrm{PlGF}_{129}$ homodimers had only little effect in this in vivo assay (Fig. 1, $D-G)$. Pellets containing sucrose aluminum sulfate without growth factors did not induce corneal neovascularization (Fig. $1 A)$.

Hypoxia effect on PlGF and VEGF $m R N A$ expression in human choriocarcinoma cell lines. The expression of PlGF and VEGF mRNAs was examined in human JAR (Fig. 2, lanes 1 and 2) and JE-3 (lanes 3 and 4) choriocarcinoma cell lines. Two major transcripts of $3.9 \mathrm{~kb}$ and $4.3 \mathrm{~kb}$, corresponding to the sizes of reported human VEGF mRNA, were present in both cell lines (Fig. 2 b, lanes 1-4). A single 1.7-kb mRNA species with expected size for human PlGF transcript $(20,23)$ was also detected in both cell lines (Fig. $2 a$, lanes 1-4). The expression levels of PIGF mRNA in these cell lines (Fig. $2 a$, lanes 1 and 3) were $\sim 10-20$-fold higher than those of VEGF (Fig. $2 b$, lanes 1 and 3). These results indicate that PIGF and VEGF are synthesized from the same tumor cells.

To investigate if the expression level of PlGF mRNA could be regulated by hypoxia, JAR (Fig. 2, $a$ and $b$; lanes 1 and 2) and JE-3 (lanes 3 and 4) tumor cells were grown under normoxic $\left(21 \% \mathrm{O}_{2}\right)$ and hypoxic conditions $\left(2 \% \mathrm{O}_{2}\right)$, and levels of PIGF and VEGF mRNAs were measured by Northern blot analysis. The steady-state expression level of VEGF mRNA (Fig. $2 b$, lanes 1 and 3) was dramatically increased within $18 \mathrm{~h}$ of growth under low oxygen tension (Fig. $2 b$, lanes 2 and 4 ). In contrast, expression levels of PIGF mRNA were indistinguishable under normoxic (Fig. $2 a$, lanes 1 and 3 ) and hypoxic (Fig. $2 a$, lanes 2 and 4 ) conditions.

To exclude the possibility that the insensitivity of the PIGF gene to hypoxia was only limited to choriocarcinoma cells, mRNA extracted from a human hepatoma cell line, hepG2, known to express PIGF (23), was used for Northern blot analy- a.

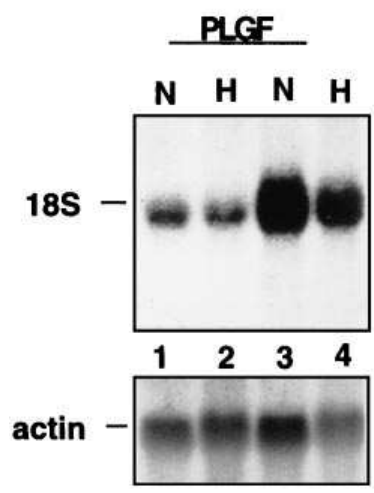

b.

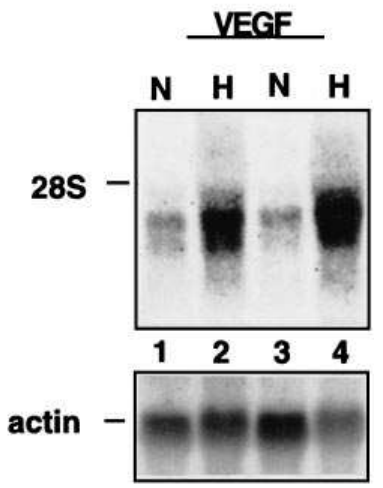

Figure 2. Hypoxia effects on mRNA expression levels of PIGF and VEGF in choriocarcinoma cell lines. Monolayers of human JAR (lanes 1 and 2) and JE-3 (lanes 3 and 4) choriocarcinoma cells were grown in $25-\mathrm{cm}^{2}$ flasks to confluency. Total RNAs were isolated from cells incubated for $18 \mathrm{~h}$ under normoxic $(\mathrm{N})$ and hypoxic $(\mathrm{H})$ conditions. The total RNAs were analyzed on the Northern blot by hybridizing with a human PIGF-2 cDNA probe ( $a$ ) (see Methods) or a hu-

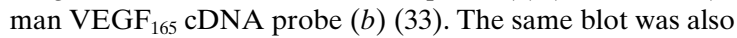
hybridized to a human actin cDNA probe ( $a$ and $b$ ).

sis. As shown in Fig. 3, the expression of PIGF mRNA in these cells was not affected by hypoxia regulation. In contrast, the level of VEGF mRNA was significantly increased by exposure to hypoxia. No evidence of cell death could be detected in cultures exposed to hypoxia, and cells continued to proliferate at a normal rate after incubation in normal oxygen tension. These data suggest that the gene structures in response to low oxygen are different between PIGF and VEGF although they share a high degree of sequence homology in their coding regions.

Elevation of heterodimerization between PlGF and VEGF by hypoxia. Three sensitive ELISA immunoassays were established using specific antibodies against PlGF, VEGF, or PlGF/VEGF heterodimers. Each assay was designed to detect a specific homodimer or heterodimer (24). Our previous results showed the natural occurrence and secretion of PlGF/ VEGF heterodimers in tumor cells (24). To study if hypoxia affected the heterodimerization between PIGF and VEGF in tumor cells, the conditioned medium derived from JE-3 cho-

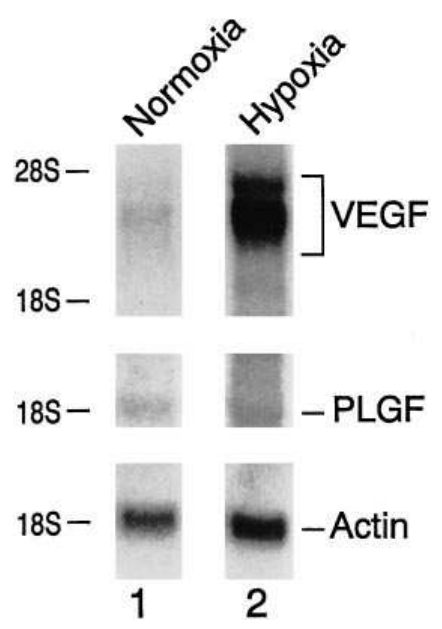

Figure 3. Hypoxia effects on mRNA expression levels of PIGF and VEGF in a human hepatoma cell line. Monolayers of human Hep G2 hepatoma cells were grown in $25-\mathrm{cm}^{2}$ flasks to confluency. Total cellular RNAs were isolated from cells incubated for $18 \mathrm{~h}$ under normoxia (lane 1 ) and hypoxia (lane 2) conditions as described in Methods The RNA were analyzed by the Northern blot analysis by using PIGF-2 and VEGF 165 cDNA probes. The migrations of $18 \mathrm{~S}$ and $28 \mathrm{~S}$ ribosomal RNA are indicated on the left as molecular markers. 


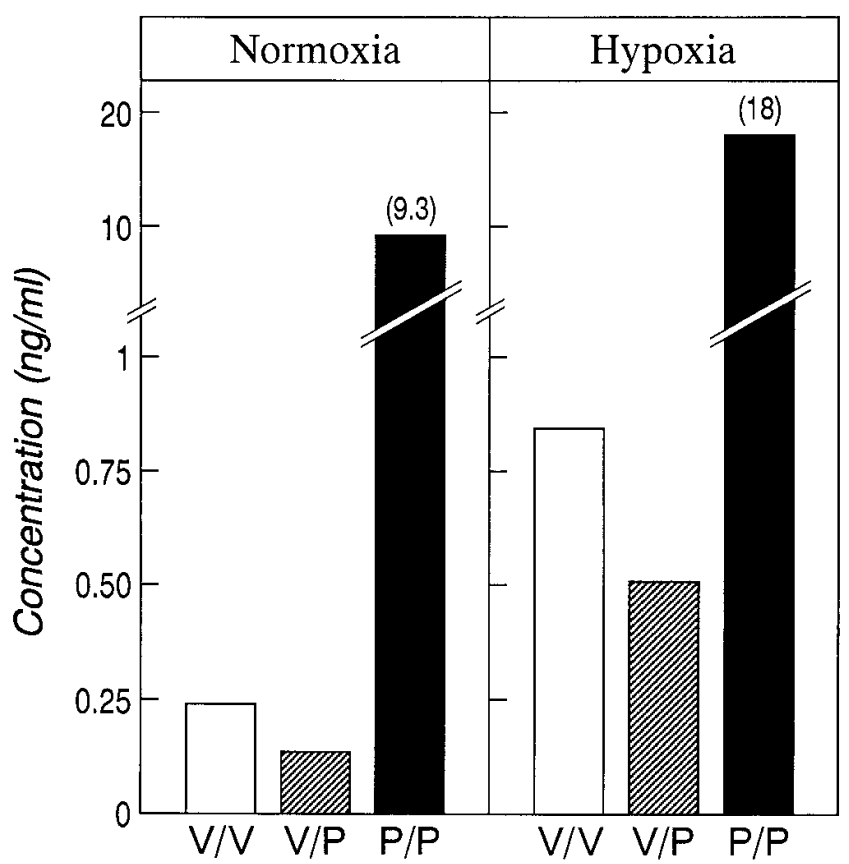

Figure 4. Hypoxia effect on protein levels of PIGF, VEGF, and $\mathrm{PlGF} / \mathrm{VEGF}$ heterodimers as detected by ELISA assays. Sensitive ELISA assays that specifically detect PIGF, VEGF and PIGF/VEGF heterodimers were developed as previously described (24). Monolayers of human JE-3 choriocarcinoma cells were grown to confluency in $60-\mathrm{cm}^{2}$ dishes. The conditioned medium was replaced with fresh medium and soluble heparin $(10 \mathrm{U} / \mathrm{ml})$ was added to cells. After $48 \mathrm{~h}$ incubation, conditioned media derived from hypoxia and normoxia were used to detect levels of PlGF, VEGF, and PIGF/VEGF heterodimers. $V / V$, VEGF homodimers; $V / P$, VEGF/PlGF heterodimers; $P / P, \mathrm{PlGF} / \mathrm{PlGF}$ homodimers.

riocarcinoma cells was analyzed by immunoassay. Soluble heparin at a concentration of $10 \mathrm{U} / \mathrm{ml}$ was added to the cells in order to release heparin-binding growth factors on the cell surface and in the extracellular matrix as sequestered by heparan sulfate proteoglycans.

In parallel with the increase in mRNA level in JE-3 cells (Fig. 2), PlGF protein levels under normoxic conditions were more than 20-fold higher than VEGF as shown in Fig. 4. The concentration of PlGF/VEGF heterodimers $(0.13 \mathrm{ng} / \mathrm{ml})$ was slightly lower than that of VEGF homodimers $(0.24 \mathrm{ng} / \mathrm{ml})$. Under hypoxic conditions, an approximately fourfold increase of VEGF homodimers was detected in the conditioned medium (Fig. 4). There was a similar fourfold increase in PlGF/ VEGF heterodimers. In contrast, the protein level of PIGF homodimers increased less than twofold under the same conditions (Fig. 4). These data demonstrated that the excess amount of PIGF in JE-3 cells heterodimerized with the increased level of VEGF protein when stimulated by hypoxia. Thus, hypoxia indirectly regulates the expression level of PIGF/VEGF heterodimers in these tumor cells.

\section{Discussion}

VEGF has mitogenic and non-mitogenic effects on endothelial cells including chemotaxis, increase of vascular permeability and activation of proteases, which are essential for angiogenesis. It is the most specific growth factor on endothelial cells (6,
12, 13, 24). The identification of VEGF-related growth factors including PlGF, VEGF-B, and VEGF-C implies a complexity and redundancy of biological functions of these angiogenic molecules. Among these angiogenic factors, at least VEGF and PIGF can exist as alternatively spliced various multi-isoforms $(5,23)$. These VEGF and PIGF isoforms together with VEGF-B and VEGF-C may form various homo- and heterodimers due to the presence of eight conserved cysteine residues in the platelet derived growth factor (PDGF)-like domain. These dimerizations could further increase the complexity of their secretion, heparin-binding affinity, receptor interaction and biological functions. The formation of different dimeric complexes suggests that cellular signals mediated by these growth factors are at least partially overlapping.

Our present work describes the in vivo angiogenic activity of $\mathrm{PlGF}_{129}$ homodimers, $\mathrm{PlGF}_{129} / \mathrm{VEGF}_{165}$ heterodimers and $\mathrm{VEGF}_{165}$ homodimers as an example of the diverse activity of VEGF family. We chose the mouse corneal neovascularization assay as our in vivo model because it has several advantages over other angiogenesis assays (27). It is a reproducible and quantitative method which permits testing the angiogenic activity of a compound in a genetically defined murine strain. The $\mathrm{VEGF}_{165}$-induced intense corneal neovascularization in $\mathrm{C} 57 \mathrm{~B} 16 / \mathrm{J}$ mice is consistent with its potent mitogenic and chemotactic activities on endothelial cells. The $\mathrm{PlGF}_{129} / \mathrm{VEGF}_{165}$ heterodimer stimulated new vessel growth with similar vessel length but with much less density. This in vivo finding is consistent with our previous in vitro work demonstrating that $\mathrm{PlGF}_{129} / \mathrm{VEGF}_{165}$ heterodimers were equally as potent as $\mathrm{VEGF}_{165}$ homodimers in stimulating endothelial cell migration, whereas the heterodimers displayed 20-50 fold less mitogenic activity than $\mathrm{VEGF}_{165}$ homodimers (24). Similar to our previous in vitro results (24), $\mathrm{PlGF}_{129}$ alone was not sufficiently potent to induce angiogenesis. Thus, the biological function of PlGF still remains obscure. In a recent report, Sawano et al. showed that PIGF-2 had growth stimulatory activity on human umbilical vein endothelial cells (28).

Two high affinity tyrosine kinase receptors, Flk-1/KDR and Flt-1, are involved in signaling in endothelial cells stimulated by VEGF and PlGF (12). Flk-1 mediated signals cause striking changes in endothelial cell morphology, VEGF-induced mitogenicity, and chemotaxis, whereas Flt-1 fails to induce such responses to VEGF (29). Both VEGF and PIGF/VEGF heterodimers bind to Flk-1/KDR receptor with a similar affinity. The fact that PlGF binds only to Flt-1, but not Flk-1, suggest that this growth factor is not able to stimulate the outgrowth of new blood vessels. The Flt- 1 mediated response in endothelial cells remains to be characterized.

However, genetically deficient mice with mutations that inactive Flt-1 receptor are embryonic lethal due to a malformation of vasculature (17), suggesting that this receptor is important in endothelial cell differentiation, vascular organization and probably maintenance of vasculature during embryonic development. Current angiogenic assays are not able to detect such in vivo effects. Mice homozygous with mutations that inactive Flk-1/KDR are also embryonic lethal due to lack of the blood island formation in yolk sac (18). However, mice heterozygous with loss of a single VEGF allele is lethal in the embryo between day 11 and $12(15,16)$. Neovascularization and blood island formation are impaired (16). These findings suggest that other ligands such PIGF, VEGF-B and VEGF-C and their heterodimers may activate Flk-1 and Flt-1 receptors. 
Our data also demonstrate that PIGF and VEGF are differentially regulated by hypoxia. Steady-state levels of VEGF mRNA and protein in both human choriocarcinoma and hepatoma cell lines were markedly increased in response to hypoxia exposure. In contrast to VEGF, the mRNA expression level of PlGF in these cell lines was not affected by hypoxic regulation. These data suggest that although PlGF and VEGF have a high sequence similarity in the coding region, their gene structures in response to oxygen are different. An increase of PlGF protein of approximately twofold was detected under hypoxic conditions. It is yet not clear whether the increase of PlGF protein is due to post-transcription regulation or due to an increase of stability of PlGF protein under hypoxia.

Recent findings also indicate that hypoxia increases VEGF mRNA stability rather than stimulating new RNA synthesis (30). Our previous results demonstrate that several tumor cell lines were able to produce PIGF and VEGF (24). In addition, these tumor cells also secrete PlGF/VEGF heterodimers into the conditioned medium. Some of these cell lines such as JAR and JE-3 choriocarcinoma cells synthesize PlGF at a level more than 20-fold higher than VEGF. Thus, hypoxia alters the ratio of protein synthesis between PIGF and VEGF in these cells. The excess amount of PlGF heterodimerizes with the VEGF molecules which are synthesized by hypoxia stimulation. It would be interesting to investigate if there are any changes in the proportion of the various isoforms of PlGF upon hypoxia induction.

Such a hypoxic situation is relevant in tumor growth. During tumor growth, increasing tumor volume results in a low oxygen environment, especially in the central area of the tumor mass (10). The elevated levels of expression of VEGF by hypoxia heterodimerizes with the excess PlGF, which participates in the regulation of the tumor angiogenesis although PlGF homodimers do not stimulate angiogenesis. Thus, hypoxia regulates not only the expression level of VEGF but also the angiogenic activity of VEGF and PIGF in tumor cells coexpressing both factors.

\section{Acknowledgments}

We thank Drs. Richard-Weidong Ji, Jack Arbiser, and Blair Marshall for their critical review of the manuscript.

Y. Cao is supported by the International Human Frontier Science Program. This work was supported in part by a grant from EntreMed, Rockville, Maryland and USHPS grant CA-45548.

\section{References} 10934.

1. Folkman, J., and Y. Shing. 1992. Angiogenesis. J. Biol. Chem. 267:10931-

2. Folkman, J. 1995. Angiogenesis in cancer, vascular rheumatoid and other disease. Nat. Med. 1:27-31.

3. Folkman, J. 1995. Clinical applications of research on angiogenesis. $N$. Engl. J. Med. 333:1757-1763.

4. Ferrara, N., and W.J. Henzel. 1989. Pituitary follicular cells secrete a novel heparin binding growth factor specific for Vascular endothelial cells. Biochem. Biophys. Res. Commun. 161:851-858.

5. Gospodarowicz, D., J.A. Abraham, and J. Schilling. 1989. Isolation and characterization of a vascular endothelial cell mitogen produced by pituitaryderived folliculo stellate cells. Proc. Natl. Acad. Sci. USA. 86:7311-7315.

6. Leung, D.W., G. Cachianes, W.J. Kuang, D.V. Goeddel, and N. Ferrara. 1989. Vascular endothelial growth factor is a secreted angiogenic mitogen. Science (Wash. DC). 246:1306-1309.

7. Senger, D.R., S.J. Galli, A.M. Dvorak, C.A. Perruzzi, V.S. Harvey, and H.F. Dvorak. 1983. Tumor cells secrete a vascular permeability factor that promotes accumulation of ascites fluid. Science (Wash. DC). 219:983-985.
8. Ferrara, N., K. Houck, L. Jakeman, J. Winer, and D.W. Leung. 1991. The vascular endothelial growth factor family of polypeptides. J. Cell. Biochem. 47: 211-218.

9. Ferrara, N., K. Houck, L. Jakeman, and D.W. Leung. 1992. Molecular and biological properties of the vascular endothelial growth factor family of proteins. Endocr. Rev. 13:18-32.

10. Shweiki, D., A. Itin, D. Soffer, and E. Keshet. 1992. Vascular endothelial growth factor induced by hypoxia may mediate hypoxia-initiated angiogenesis. Nature (Lond.). 359:843-845.

11. Shima, D.T., A.P. Adamis, N. Ferrara, K.-T. Yeo, P. Allende, J. Folkman, and P.A. D'Amore. 1995. Hypoxic induction of endothelial cell growth factors in retinal cells: Identification and characterization of vascular endothelial growth factor (VEGF) as the mitogen. Mol. Med. 1:182-193.

12. Mustonen, T., and K. Alitalo. 1995. Endothelial receptor tyrosine kinases involved in angiogenesis. J. Cell Biol. 129:895-898.

13. Thomas, K. 1996. Vascular endothelial growth factor, a potent and selective angiogenic agent. J. Biol. Chem. 271:603-606.

14. Shibuya, M. 1995. Role of VEGF-flt receptor system in normal and tumor angiogenesis. Adv. Cancer Res. 67:281-316.

15. Carmeliet, P., V. Ferrara, G. Breier, S. Pollefeyt, L. Kiechens, M. Gertsenstein, M. Fahrig, A. Vandenhoek, K. Harpal, C. Eberhardt et al. 1996. Abnormal blood vessel development and lethality in embryos lacking a single VEGF allele. Nature (Lond.). 380:435-439.

16. Ferrara, N., K. Carver-Moore, H. Chen, M. Dowd, L. Lu, K. S. O'Shea, L. Powell-Braxton, K.J. Hillan, and M.W. Moore. 1996. Heterozygous embryonic lethality induced by targeted inactivation of the VEGF gene. Nature (Lond.). 380:439-442.

17. Fong, G.-H., J. Rossant, M. Gertsenstein, and M.L. Breitman. 1995. Role of the Flt-1 receptor tyrosine kinase in regulating the assembly of vascular endothelium. Nature (Lond.). 376:66-70.

18. Shalaby, F., J. Rossant, T.P. Yamaguchi, M. Gertsenstein, X.-F. Wu, M.L. Breitman, and A.C. Schuh. 1995. Failure of blood-island formation and vasculogenesis in Flk-1-deficient mice. Nature (Lond.). 376:62-66.

19. Sato, T.N., Y. Tozawa, U. Deutsch, K. Wolburg-Buchholz, Y. Fujiwara, M. Gendron-Maguire, T. Gridley, H. Wolburg, W. Risau, and Y. Qin. 1995. Distinct roles of receptor tyrosine kinases Tie- 1 and Tie- 2 in blood vessel formation. Nature (Lond.). 376:70-74.

20. Maglione, D., V. Guerriero, G. Viglietto, P. Delli-Bovi, and M.G. Persico. 1991. Isolation of a human placenta cDNA coding for a protein related to the vascular permeability factor. Proc. Natl. Acad. Sci. USA. 88:9267-9271.

21. Olofsson, B., K. Pajusola, A. Kaipainen, G. von Euler, V. Joukov, O. Saksela, A. Orpana, R.F. Pettersson, K. Alitalo, and U. Eriksson. 1996. Vascular endothelial growth factor B, a novel growth factor for endothelial cells. Proc. Natl. Acad. Sci. USA. 93:2576-2581.

22. Joukov, V., K. Pajusola, A. Kaipainen, D. Chilov, I. Lahtinen, E. Kukk, O. Saksela, N. Kalkkinen, and K. Alitalo. 1996. A novel vascular endothelial growth factor, VEGF-C, is a ligand for the Flt4 and KDR (VEGFR-2) receptor tyrosine kinases. EMBO (Eur. Mol. Biol. Organ.) J. 15:290-298.

23. Maglione, D., V. Guerriero, G. Viglietto, M.G. Ferraro, O. Aprelikova, K. Alitalo, S.D. Vecchio, K.-J. Lei, J.Y. Chou, and M.G. Persico. 1993. Two alternative mRNAs coding for the angiogenic factor, placenta growth factor (PlGF), are transcribed from a single gene of chromosome 14. Oncogene. 8: 925-931.

24. Cao, Y., H. Chen, L. Zhou, M.-K. Chiang, B. Anand-Apte, J.A. Weatherbee, Y. Wang, F. Fang, J.G. Flanagan, and M.L.-S. Tsang. 1996. Heterodimers of placenta growth factor/vascular endothelial growth factor: endothelial activity, tumor cell expression, and high affinity binding to Flk-1/KDR. $J$. Biol. Chem. 271:3154-3162.

25. DiSalvo, J., M.L. Bayne, G. Conn, P.W. Kwok, P.G. Trivedi, D.D. Soderman, T.M. Palisi, K.A. Sullivan, and K.A. Thomas. 1995. Purification and characterization of a naturally occurring vascular endothelial growth factor-placenta growth factor heterodimer. J. Biol. Chem. 270:7717-7723.

26. Park, J.E., H.E. Chen, J. Winer, and N. Ferrara. 1994. Placenta growth factor: potentiation of vascular endothelial growth factor bioactivity in vitro and in vivo and high affinity binding to Flt-1 but not to Flk-1/KDR. J. Biol. Chem. 269:25646-25654.

27. Cao, Y., C. Chen, J.A. Weatherbee, M. Tsang, and J. Folkman. 1995. gro- $\beta$, a -C-X-C- chemokine, is an angiogenesis inhibitor that suppresses the growth of Lewis lung carcinoma in mice. J. Exp. Med. 182:2067-2077.

28. Sawano, A., T. Takahashi, S. Yamaguchi, M. Aonuma, and M. Shibuya. 1996. Flt-1 but not KDR/Flk-1 tyrosine kinase is a receptor for placenta growth factor, which is related to vascular endothelial growth factor. Cell Growth Differ. 7:213-221.

29. Waltenberger, J., L. Claesson-Welsh, A. Siegbahn, M. Shibuya, and C.H. Heldin. 1994. Different signal transducting properties of KDR and Flt-1, two receptors for vascular endothelial growth factor. J. Biol. Chem. 269:2698826995.

30. Shima, D.T., U. Deutch, and P.A. D'Amore. 1995. Hypoxia induction of vascular endothelial growth factor (VEGF) in human epithelial cells is mediated by increases in mRNA stability. FEBS lett. 370:203-208.

31. Kenyon, B.M., E.E. Voest, C. Chen, E. Flynn, J. Folkman, and R.J. D'Amato. 1996. A model of angiogenesis in the mouse cornea. Invest. Ophthal. Visual Sci. 37:1625-1632. 